David Sebastián Flores-Romero; Hugo Jaime Avendaño-Fajardo; Mireya Magdalena Torres-Palacios

http://dx.doi.org/10.35381/r.k.v5i3.906

\title{
Planificación estratégica situacional del Instituto de Fomento al Talento Humano
}

\section{Situational strategic planning of the institute for the promotion of human talent}

\author{
David Sebastián Flores-Romero \\ david.flores.67@psg.ucacue.edu.ec \\ Universidad Católica de Cuenca, Cuenca \\ Ecuador \\ https://orcid.org/0000-0003-0418-5113 \\ Hugo Jaime Avendaño-Fajardo \\ hugo.avendano@ucacue.edu.ec \\ Universidad Católica de Cuenca, Cuenca \\ Ecuador \\ https://orcid.org/0000-0001-9206-5052 \\ Mireya Magdalena Torres-Palacios \\ mireya.torres@ucacue.edu.ec \\ Universidad Católica de Cuenca, Cuenca \\ Ecuador \\ https://orcid.org/0000-0002-7724-3313
}

Recibido: 20 de mayo de 2020

Revisado: 15 de junio de 2020

Aprobado: 30 de julio de 2020

Publicado: 15 de agosto de 2020 


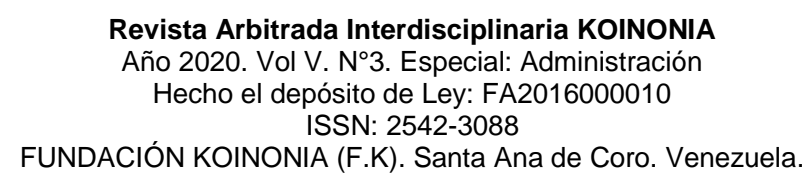

David Sebastián Flores-Romero; Hugo Jaime Avendaño-Fajardo; Mireya Magdalena Torres-Palacios

\title{
RESUMEN
}

La investigación tiene por objetivo analizar la planificación estratégica situacional (PES) del Instituto de Fomento al Talento Humano del Ecuador. La metodología se basó en un tipo descriptiva con diseño no experimental de campo, siendo la muestra poblacional conformada por 269 becarios del Instituto de Fomento al Talento Humano del Ecuador. La PES ayudó a fijar prioridades, permitiendo concentrarse en las fortalezas y debilidades del instituto, ayudando a solventar los problemas de cambios en el entorno interno y externo, depende de ella que se logren los objetivos organizacionales con éxito. El IFTH en un tiempo de 10 meses puede mejorar todos los servicios en cuanto a becas para poder satisfacer las necesidades de los becarios que se encuentran al momento inconformes por temas de insatisfacción de los becarios.

Descriptores: Planificación estratégica; método de planificación; toma de decisiones; operación administrativa. (Tomadas del Tesauro UNESCO).

\begin{abstract}
The research aims to analyze the situational strategic planning (SSP) of the Institute for the Promotion of Human Talent of Ecuador. The methodology was based on a descriptive type with a non-experimental field design, with the population sample made up of 269 scholars from the Institute for the Promotion of Human Talent of Ecuador. The SSP helped to set priorities, allowing to focus on the strengths and weaknesses of the institute, helping to solve the problems of changes in the internal and external environment, it depends on it that the organizational objectives are successfully achieved. The IFTH in a period of 10 months can improve all the services in terms of scholarships in order to satisfy the needs of the scholarship recipients who are currently dissatisfied due to issues of dissatisfaction of the scholarship recipients.
\end{abstract}

Descriptors: Strategic planning; planning methods; decision making; management operations. (Taken from the UNESCO Thesaurus). 


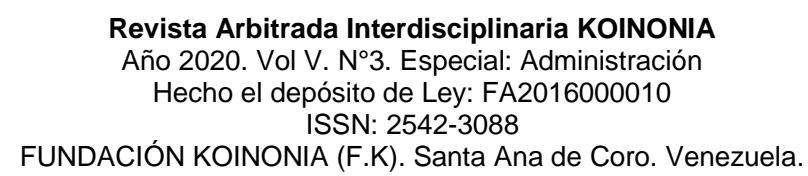

David Sebastián Flores-Romero; Hugo Jaime Avendaño-Fajardo; Mireya Magdalena Torres-Palacios

\section{INTRODUCCIÓN}

El plan táctico se concibe como una implementación de progresos sistemáticos para lograr los propósitos y metas del establecimiento, siendo un procedimiento que yace de "la toma anticipada de decisiones consignadas a reducir la incertidumbre y las sorpresas, a guiar a la acción hacia una situación anhelada, mediante una instrumentación reflexiva de medios" (Ossorio, 2003), siendo necesario por parte de la organización, fijar las metas a conseguir durante un periodo de tiempo determinado, situación contribuyente a generar una ruta de trabajo sistemático con la finalidad de ser efectivos en el cumplimiento de los proposositos diseñados (Ávila, et al., 2019), si no se tiene claridad en la gestión organizacional, se corre el riesgo de asignar recursos de forma caprichosa, pudiendo perjudicar los fines organizativos (Armijo, 2011).

En función de lo planteado, se aborda la planificación estratégica situacional del Instituto de Fomento al Talento Humano del Ecuador (IFTH), mediante la Planificación Táctica del IFTH para el periodo 2020-2023, asentada en el método de SENPLADES y afín a la regla que acoge al IFTH, basada en la Carta Magna, el "PNBV" conocido como Plan Nacional del Buen Vivir, las estrategias internas de los sectores del Gobierno Coordinador de Capacidad Humana y el método céntrico del Organismo. Este se aceptó con las autoridades que conformaron la sesión, así como las máximas mandos del establecimiento, para luego ser presentado al ente rector (Instituto de Fomento al Talento Humano, 2016).

El experimento voluntario de abastecer o seleccionar medios, que asignándolos coherentemente lleva a las conclusiones deseadas, traduciéndose en el asunto mediante el cual se pronostica el futuro, desde el presente con base en el pasado, donde no sólo se intenta pronosticar lo que viene más adelante (Argudo-Tello, et al., 2019), sino además, labrar el porvenir, transformando un futuro esperado en un futuro deseado (Gutierrez, et al., 2016). La planificación es un método muy importante en varios establecimientos e instituciones públicas por cuanto se ha convertido en una herramienta primordial (Armijo, 2011). 


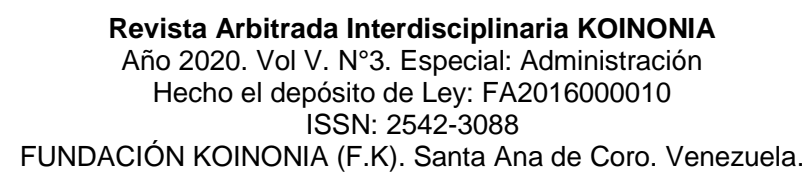

David Sebastián Flores-Romero; Hugo Jaime Avendaño-Fajardo; Mireya Magdalena Torres-Palacios

El IFTH promueve el ingreso igualitario para la ciudadanía a proyectos de refuerzo de capacidad humano, de acuerdo con las directrices emitidas por autoridades máximas; a través de la administración de créditos educativos, otorgamiento y administración de becas, ayudas económicas (Reyes, et al., 2020). Su misión está colaborando con el crecimiento educativo de los beneficiarios, con ayuda de asistencia técnica, su guía metodológica, colaboración presupuestaria y control de cumplimiento del objetivo (Rivera, 2011).

La visión del IFTH hacia el año 2023 es prestar servicios de perfección, darse a conocer para tener profesionales con calidad humana y profesional, que aporten al adelanto académico de los becarios, para lo cual se divide en: 4 áreas ejecutoras: Becas, Créditos, Coactivas, Relacionamiento Interinstitucional. 7 áreas de apoyo: Financiera, Administrativa, Talento Humano, Jurídica, TIC, Planificación, Comunicación Social. Los valores de la Institución son: El enfoque estratégico, la ética, la efectividad, la transparencia, la imparcialidad, el trabajo en equipo, la honestidad, la responsabilidad, la calidez y el enfoque al ciudadano (Instituto de Fomento al Talento Humano, 2020).

Por otro lado, el Plan Táctico Situacional nace del mejoramiento de una planificación, la cual se plantea diferentes compendios a su proyecto al conceptuarla como una secuencia para lograr situaciones, metas, conocer la capacidad de oponentes que se varían con tiempos críticos, siempre bajo condiciones de riesgo (Salazar \& Romero, 2006). La planeación táctica toma el nombre de planificación tradicional, para solucionar dificultades más complicadas, siendo así un instrumento que puede llegar a conducir un proceso en todas sus dimensiones (Gutiérrez, et al., 2016).

En este sentido, se debe realizar un recorte problemático de la realidad social. Este recorte contendrá varios factores: la explicación es auto-referencial, es siempre la explicación de un actor; es dinámica, artículo pasado, presente y futuro; tiene varios centros importantes por cuanto se tomaron datos de todos ellos; es totalizadora, porque al tomar estos datos, estos deben unirse al momento de definir causas y problemas importantes; es rigurosa, en el sentido de que es una explicación que supera las causas 
sintomáticas y procura discernir causas más esenciales, etc (Calle-Abril, et al., 2020). La característica esencial es el policentrismo por cuanto se habla de un enfoque comunicativo (Criollo-Aguirre, et al., 2019). A partir de autores del enfoque hermenéutico el cual se basa en diálogos entre los entes implicados al momento de entender y superar los problemas encontrados. Estos diálogos harán que los problemas sean iguales de importantes para todos los entes involucrados, lo que logrará generar que la solución a estos se articulen en absoluto en la explicación de un único actor (Rivera, 2011).

La planificación estrategica situacional (PES), se encuentra presente en todo tipo de investigación y proyectos de alta dirección Es una metodología aplicada en ámbitos gubernamentales, sociedades, sistemas públicos, con el fin de lograr mejores resultados en los procesos que se llevan a cabo. Como por ejemplo en elaboraciones de planes de Gobierno o también en campañas electorales (Salazar \& Romero, 2006), en la planificación pública en la mayoría de los casos estará presente la planificación estratégica situacional, como un nuevo instrumento que logrará niveles de gobernabilidad y construir viabilidad al cambio, es decir este instrumento ayudará a conseguir logros gubernamentales y sociales, por cuanto contribuirá en solucionar problemas de manera eficaz (Salazar \& Romero, 2006).

\section{Referencial Teórico}

\section{La Planificación estratégica situacional en el sector público}

Para la elaboración de la planificación estratégica situacional del Instituto de Fomento al Talento Humano, se toman en cuenta las etapas subsiguientes:

1. Estudios de las herramientas de planificación: con el fin de modular el Plan Estratégico Institucional del Instituto de Fomento al Talento Humano a la planificación nacional, se analizan las normativas procedentes de la Constitución, del Plan Nacional del Buen Vivir, de las Agendas y Políticas Sectoriales, como las Coordinaciones Zonales; para sintetizar en los objetivos estratégicos, estrategias e indicadores de la institución. 
2. Análisis situacional: Se realiza un análisis de medio macro y micro del instituto, revisando las necesidades de estrategias acorde la propuesta de la CEPAL ILPES, la cual indica que "Para surgir del atasco se requieren innovaciones, nuevos modelos intelectuales y destrezas organizativas que fortifiquen las entidades. No se puede salir del círculo vicioso con el mismo comportamiento y nivel de pensamiento que genera el estancamiento dentro del círculo vicioso" (Instituto latinoamericano y del caribe de planificación económica y social, 2006).

3. Diagnóstico institucional: Se revisan las aptitudes, facultades y los servicios concedidos al instituto al igual que la demanda de los servicios, la representación institucional y las circunstancias de mejorar. Es un punto de muy importante y suministra los antecedentes necesarios para el trazado del direccionamiento estratégico.

4. Descripción de direccionamiento estratégico: se analiza la capacidad del instituto para pronosticar y solucionar de mejor manera los problemas complicados de manera individual y colectiva para tomar decisiones estratégicas con autonomía y responsabilizarse por los resultados en el futuro.

5. Delineación de estrategias: establecer una orientación para alcanzar el objetivo estratégico institucional propuesto, se identifican las estrategias para alcanzar los objetivos estratégicos institucionales. De igual forma se identifican los servicios que brindan el instituto, gestiones, eventos y planes propuestos para el cumplimiento de los objetivos y la política pública.

6. Metodología de la prospectiva estratégica: es otro punto estimado para la realización de la planificación estratégica situacional que es la proyección hacia dónde se quiere llegar. En el punto de vista de la prospectiva, es importante destacar a su historia, cultura organizacional, imagen corporativa, para saber a dónde se quiere llegar con una identidad específica (Instituto de Fomento al Talento Humano, 2016). La planificación estratégica situacional como valor estratégico del Instituto, permite: 
Revista Arbitrada Interdisciplinaria KOINONIA

Año 2020. Vol V. N³. Especial: Administración

Hecho el depósito de Ley: FA2016000010

ISSN: 2542-3088

FUNDACIÓN KOINONIA (F.K). Santa Ana de Coro. Venezuela.

David Sebastián Flores-Romero; Hugo Jaime Avendaño-Fajardo; Mireya Magdalena Torres-Palacios

Definir de recursos para alcanzar las metas

Definir metas: Instrumentos para llegar a los objetivos

Generar Objetivos institucionales: marca el rumbo y la

velocidad hacia donde y como llegar

Contar con la visión institucional: Identifica grandes

prioridades a largo plazo

Determinar la imagen de la instución

Figura 1. Prospectiva Estratégica.

Fuente: Instituto de Fomento al Talento Humano (2016). 
David Sebastián Flores-Romero; Hugo Jaime Avendaño-Fajardo; Mireya Magdalena Torres-Palacios

El tipo de plan estratégico situacional contempla la elaboración de herramientas con visión de futuro, centrado en ideas en el contexto en el que se encuentra la institución:

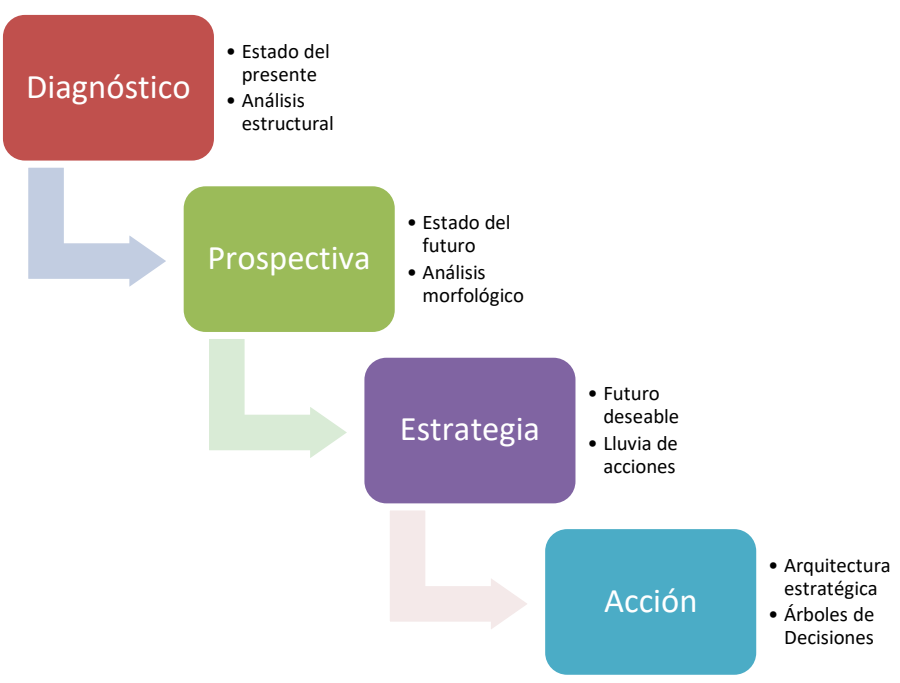

Figura 2. Modelo de planificación estratégica.

Fuente: Instituto de Fomento al Talento Humano (2016). 


\section{Descripción y diagnóstico institucional}

\section{Histórico del Instituto de Fomento al Talento Humano}

El Instituto de fomento al talento humano a través del tiempo cuenta con el siguiente proceso histórico:

\section{Histórico del IFTH}

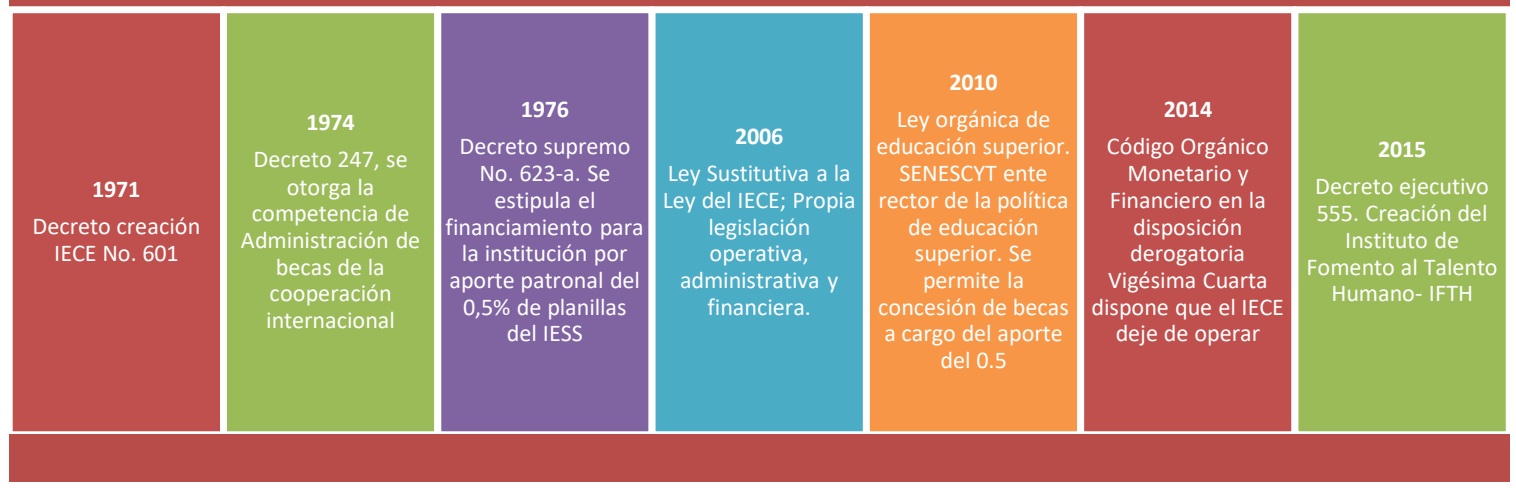

Figura 3. Antecedentes históricos del IFTH.

Fuente: Instituto de Fomento al Talento Humano (2016).

En razón de lo planteado, la investigación tiene por objetivo analizar la planificación estratégica situacional del Instituto de Fomento al Talento Humano del Ecuador.

\section{METODOLOGÍA}

La metodología se basó en un tipo descriptiva con diseño no experimental de campo, siendo la muestra poblacional conformada por 269 becarios del Instituto de Fomento al Talento Humano del Ecuador, a quienes se les aplicó encuesta y cuestionario en escala de Likert de cinco alternativas de repuesta, siendo validado por juicio de expertos y calculo de coeficiente Alfa de Cronbach con resultado de 0,79 siendo considerado confiable. Para el análisis de los datos recopilados se empleó estadistica descriptiva. 


\section{RESULTADOS}

Los resultados se presentan a continuación:

\section{Tabla 1.}

Resultados en porcentajes de las preguntas de opción múltiple de las encuestas aplicadas.

\begin{tabular}{|c|c|c|c|c|c|c|}
\hline Preguntas & $\begin{array}{c}\text { Muy } \\
\text { Bueno }\end{array}$ & Bueno & Regular & Malo & $\begin{array}{l}\text { Muy } \\
\text { Malo }\end{array}$ & Total \\
\hline $\begin{array}{l}\text { Cuál es su nivel de satisfacción con la } \\
\text { Coordinación Zonal } 6 \text { del IFTH }\end{array}$ & $18,8 \%$ & $22,5 \%$ & $28,7 \%$ & $18,1 \%$ & $11,9 \%$ & $100 \%$ \\
\hline Cómo le parecen los servicios que brinda el IFTH & $20,6 \%$ & $20,0 \%$ & $30,6 \%$ & $20,0 \%$ & $8,8 \%$ & $100 \%$ \\
\hline $\begin{array}{l}\text { Al momento de realizar la firma del contrato de } \\
\text { financiamiento cómo es la atención de los } \\
\text { servidores }\end{array}$ & $26,3 \%$ & $18,1 \%$ & $23,1 \%$ & $23,8 \%$ & $8,8 \%$ & $100 \%$ \\
\hline $\begin{array}{l}\text { Al momento de realizar sus desembolsos que tal } \\
\text { le parece la atención de los servidores }\end{array}$ & $16,3 \%$ & $21,9 \%$ & $30,0 \%$ & $22,5 \%$ & $9,4 \%$ & $100 \%$ \\
\hline $\begin{array}{l}\text { Cómo califica la eficiencia y eficacia en el } \\
\text { servicio prestado }\end{array}$ & $16,3 \%$ & $25,6 \%$ & $23,8 \%$ & $25,0 \%$ & $9,4 \%$ & $100 \%$ \\
\hline $\begin{array}{l}\text { Cuáles son las condiciones de los empleados al } \\
\text { momento de brindar el servicio }\end{array}$ & $21,9 \%$ & $18,1 \%$ & $25,0 \%$ & $26,3 \%$ & $8,8 \%$ & $100 \%$ \\
\hline $\begin{array}{l}\text { Cómo evalúa Usted el trabajo que realiza el área } \\
\text { de Becas de la Coordinación Zonal } 6 \text { del IFTH }\end{array}$ & $20,6 \%$ & $21,2 \%$ & $24,4 \%$ & $22,5 \%$ & $11,3 \%$ & $100 \%$ \\
\hline Cómo valora el profesionalismo de los servidores & $21,9 \%$ & $18,8 \%$ & $26,9 \%$ & $21,9 \%$ & $10,6 \%$ & $100 \%$ \\
\hline Cómo valora la calidad del trato a los becarios & $23,1 \%$ & $19,4 \%$ & $25,0 \%$ & $21,3 \%$ & $11,3 \%$ & $100 \%$ \\
\hline $\begin{array}{l}\text { Al momento de resolver sus dudad o inquietudes } \\
\text { cual es el comportamiento de los servidores }\end{array}$ & $19,4 \%$ & $19,4 \%$ & $30,6 \%$ & $21,3 \%$ & $9,4 \%$ & $100 \%$ \\
\hline $\begin{array}{l}\text { Considera Usted que es adecuado el tiempo en el } \\
\text { que el servidor resuelve sus inquietudes, dudas } \\
\text { o desembolsos de manera online por la } \\
\text { plataforma PUSAK o mediante Correo electrónico }\end{array}$ & $12,5 \%$ & $21,9 \%$ & $33,8 \%$ & $20,6 \%$ & $11,3 \%$ & $100 \%$ \\
\hline
\end{tabular}

Fuente: Encuesta aplicada a Becarios del IFTH. 


\section{Tabla 2.}

Resultados en porcentajes de la pregunta de opción "si o no" de las encuestas aplicadas.

\begin{tabular}{cccc}
\hline Pregunta & Si & No & Total \\
\hline $\begin{array}{c}\text { Recomendaría Usted a la } \\
\text { Coordinación Zonal 6 a } \\
\text { los demás becarios }\end{array}$ & $45,6 \%$ & $54,4 \%$ & $\mathbf{1 0 0 \%}$ \\
\hline
\end{tabular}

Fuente: Encuesta aplicada a Becarios del IFTH.

\section{PROPUESTA}

La propuesta se enmarca en mejorar la planificación estratégica situacional del IFTH por medio del método altadir de planificación popular como se detalla en la figura 5, la cual nos indica el esquema de propuesta de la presente investigación:

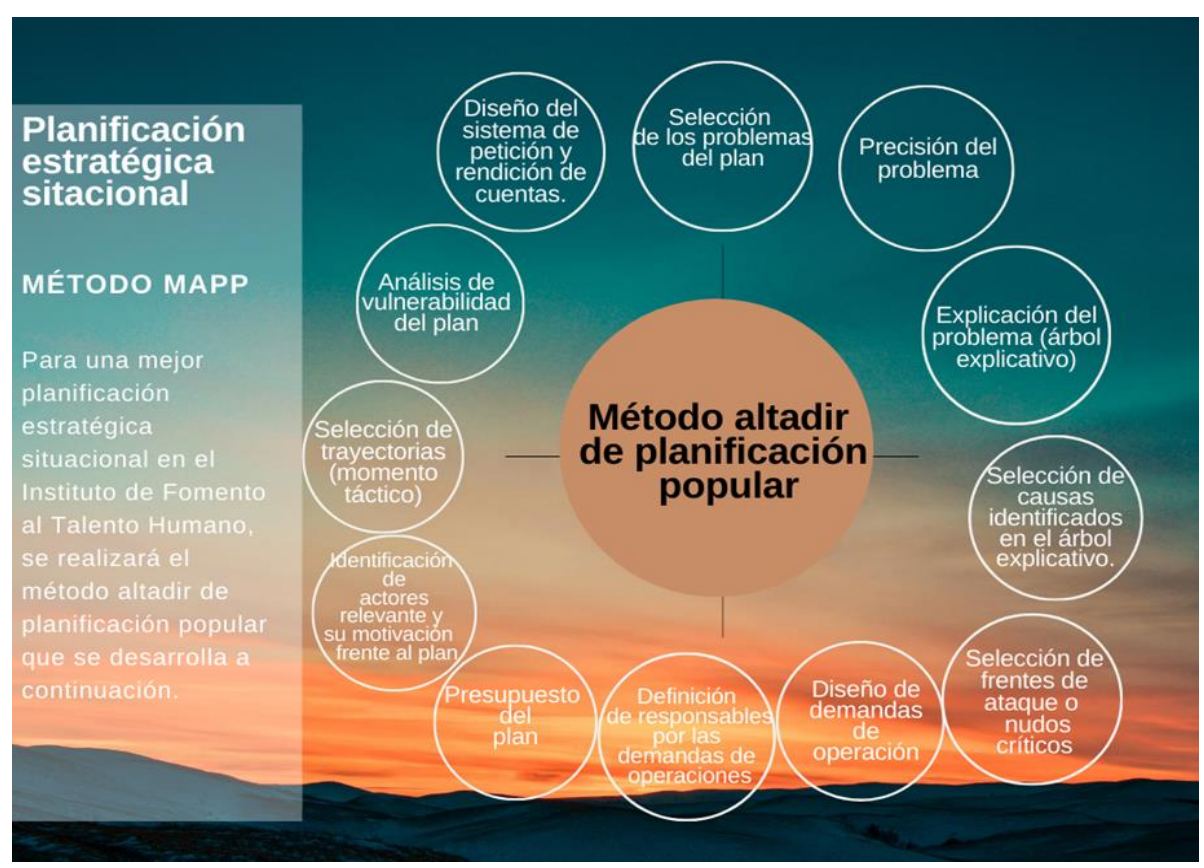

Figura 4. Esquema de propuesta método MAPP.

Fuente: Elaboración propia. 


\section{APLICACIÓN DEL MAPP}

Paso 1: Selección de los problemas del plan.- Luego de la tabulación de datos obtenidos en las encuestas se pueden observar los principales problemas de tipo administrativos y financieros que tienen los becarios y que están ligados directamente a la insatisfacción:

Problema 1.- Atención al momento de la firma de contrato.

Problema 2.- Atención al momento de recibir los desembolsos

Problema 3.- Eficiencia y eficacia en los servicios brindados

Problema 4.- Los tiempos en resolver dudas, inquietudes o desembolsos de manera online.

\section{Paso 2: Precisión del problema}

\section{Problema 1}

Nombre del problema: Atención al momento de la firma de contrato. (P1)

Actor: Analista de Becas

\section{Descripción:}

\section{Descriptores}

D1. Mala atención por parte de los El $23,1 \%$ de los becarios califican de analistas de becas

\section{Indicadores}

manera regular la atención al momento de la firma del contrato de beca 
David Sebastián Flores-Romero; Hugo Jaime Avendaño-Fajardo; Mireya Magdalena Torres-Palacios

\section{Problema 2}

Nombre del problema: Atención al momento de recibir los desembolsos. (P2)

Actor: Analista de Becas

\section{Descripción:}

\section{Descriptores}

D2. Pocos analistas para la cantidad de El $30 \%$ de los becarios califican de manera becarios

\section{Indicadores} regular la atención al momento de recibir sus desembolsos

\section{Problema 3}

Nombre del problema: Eficiencia y eficacia en los servicios brindados. (P3)

Actor: Analista de Becas

\section{Descripción:}

\section{Descriptores}

D3. No satisface las necesidades de los El $25 \%$ de los becarios califican de manera becarios

\section{Indicadores} mala la eficiencia y eficacia en los servicios brindados 
David Sebastián Flores-Romero; Hugo Jaime Avendaño-Fajardo; Mireya Magdalena Torres-Palacios

\section{Problema 4}

Nombre del problema: Los tiempos en resolver dudas, inquietudes o desembolsos de manera online. (P4)

Actor: Analista de Becas

\section{Descriptores}

D4. Respuestas tardías

$(P)=$ Problema,$(D)=$ Descriptor

\section{Descripción:}

\section{Indicadores}

El $33,8 \%$ de los becarios califican de manera regular los tiempos en resolver dudas, inquietudes o desembolsos de manera online

\section{Paso 3: Explicación del problema (árbol explicativo)}

Nombre del problema: Atención al momento de la firma de contrato. (P1)

Actor: Analista de Becas

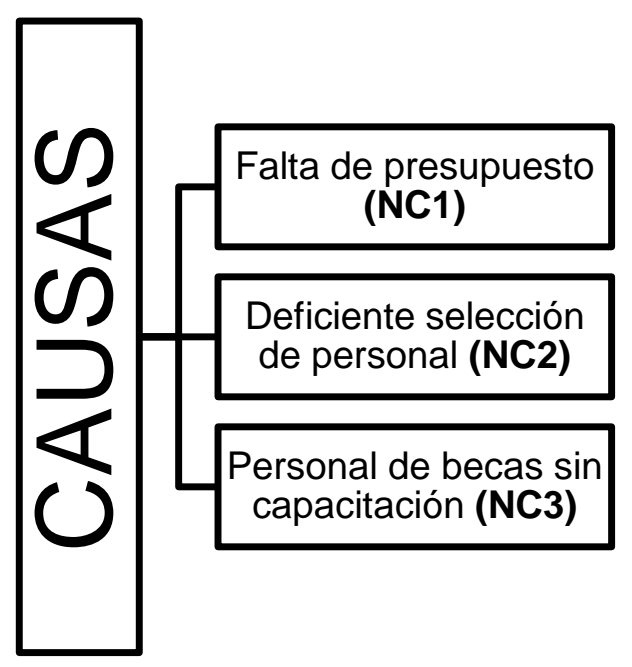


Revista Arbitrada Interdisciplinaria KOINONIA

Año 2020. Vol V. №3. Especial: Administración

Hecho el depósito de Ley: FA2016000010

ISSN: 2542-3088

FUNDACIÓN KOINONIA (F.K). Santa Ana de Coro. Venezuela.

David Sebastián Flores-Romero; Hugo Jaime Avendaño-Fajardo; Mireya Magdalena Torres-Palacios

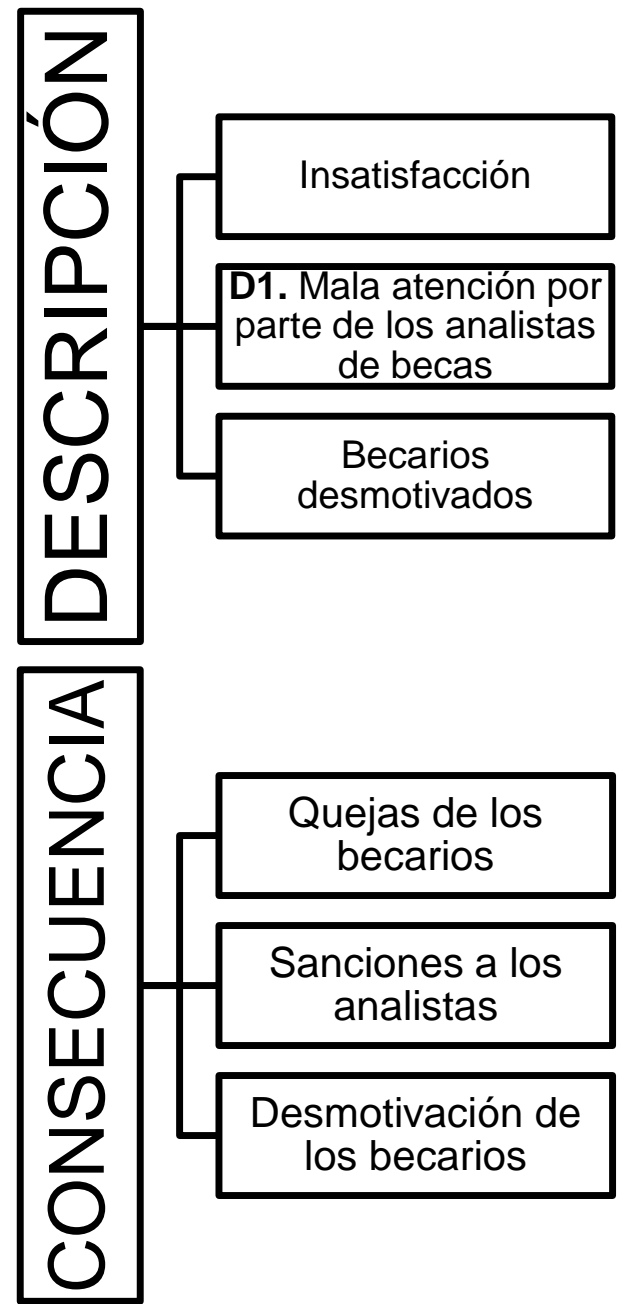

Figura 5. Explicación del problema (árbol explicativo).

Nota: $(P)=$ Problema, $(D)=$ Descriptor, $(N C)=$ Nudo Crítico . 
Revista Arbitrada Interdisciplinaria KOINONIA

Año 2020. Vol V. №3. Especial: Administración

Hecho el depósito de Ley: FA2016000010

ISSN: 2542-3088

FUNDACIÓN KOINONIA (F.K). Santa Ana de Coro. Venezuela.

David Sebastián Flores-Romero; Hugo Jaime Avendaño-Fajardo; Mireya Magdalena Torres-Palacios

Nombre del problema: Atención al momento de recibir los desembolsos. (P2)

Actor: Analista de Becas

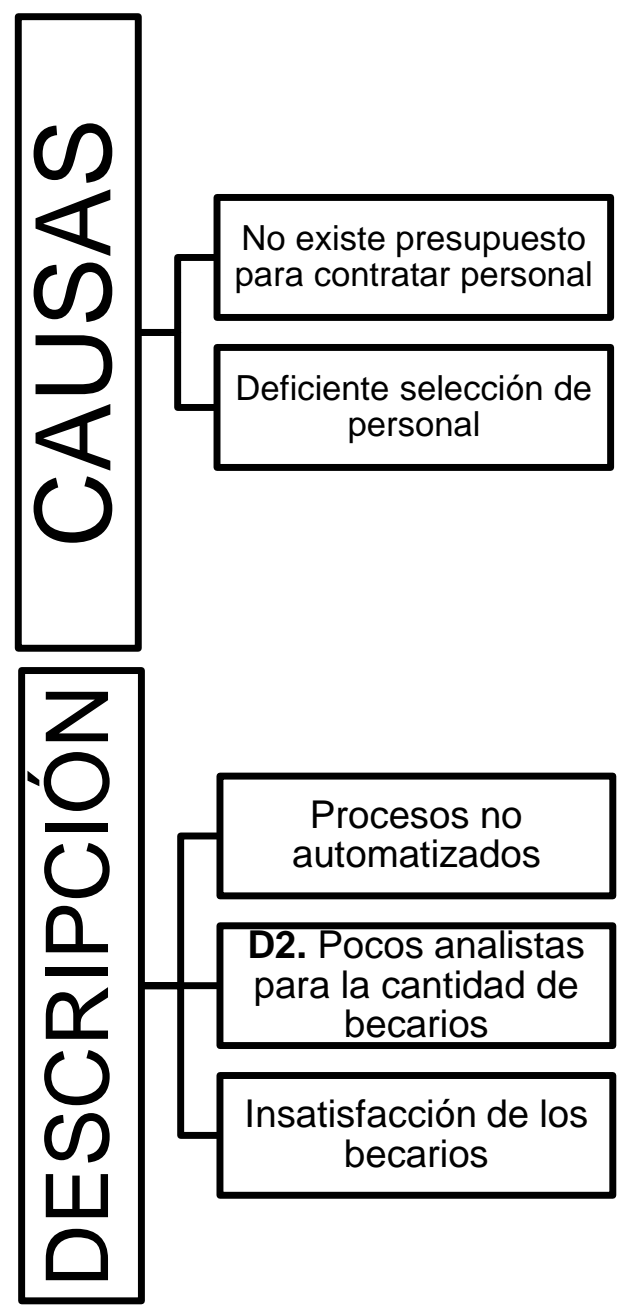


Revista Arbitrada Interdisciplinaria KOINONIA

Año 2020. Vol V. №3. Especial: Administración

Hecho el depósito de Ley: FA2016000010

ISSN: 2542-3088

FUNDACIÓN KOINONIA (F.K). Santa Ana de Coro. Venezuela.

David Sebastián Flores-Romero; Hugo Jaime Avendaño-Fajardo; Mireya Magdalena Torres-Palacios

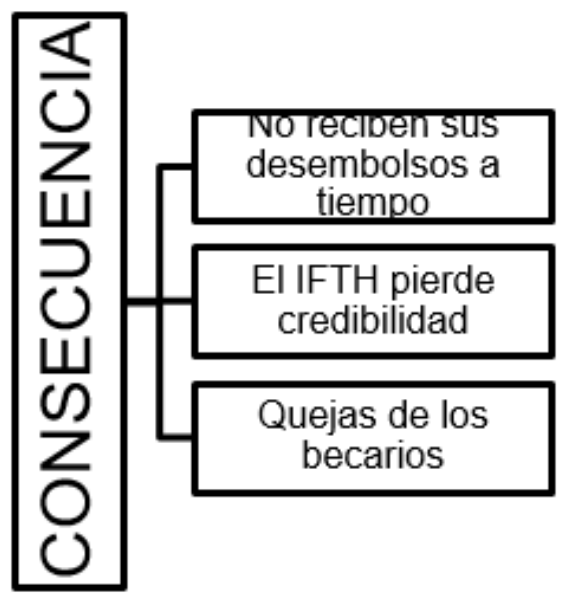

Figura 6. Atención al momento de recibir los desembolsos. (P2). Nota: $(P)=$ Problema, $(\mathrm{D})=$ Descriptor, $(\mathrm{NC})=$ Nudo Crítico

Nombre del problema: Eficiencia y eficacia en los servicios brindados. (P3)

Actor: Analista de Becas

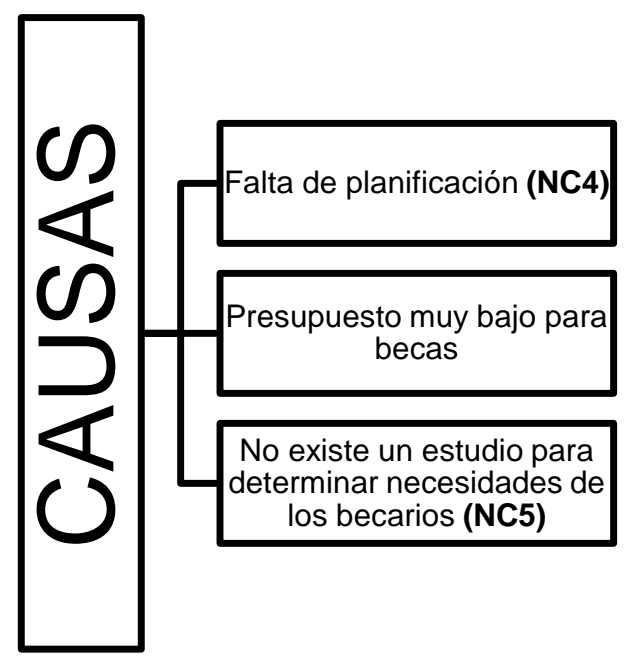


Revista Arbitrada Interdisciplinaria KOINONIA

Año 2020. Vol V. №3. Especial: Administración

Hecho el depósito de Ley: FA2016000010

ISSN: 2542-3088

FUNDACIÓN KOINONIA (F.K). Santa Ana de Coro. Venezuela.

David Sebastián Flores-Romero; Hugo Jaime Avendaño-Fajardo; Mireya Magdalena Torres-Palacios

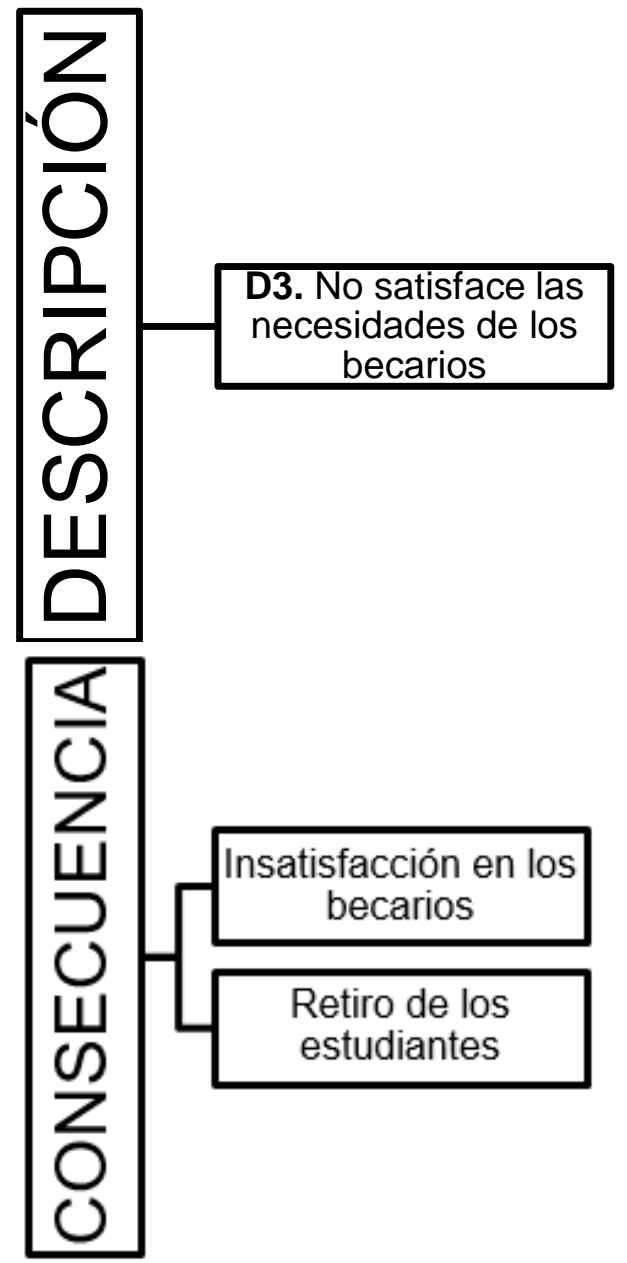

Figura 7. Eficiencia y eficacia en los servicios brindados. $(P 3)$ Nota: $(P)=$ Problema, $(D)=$ Descriptor, $(\mathrm{NC})=$ Nudo Crítico. 
David Sebastián Flores-Romero; Hugo Jaime Avendaño-Fajardo; Mireya Magdalena Torres-Palacios

Nombre del problema: Los tiempos en resolver dudas, inquietudes o desembolsos de manera online. (P4)

Actor: Analista de Becas

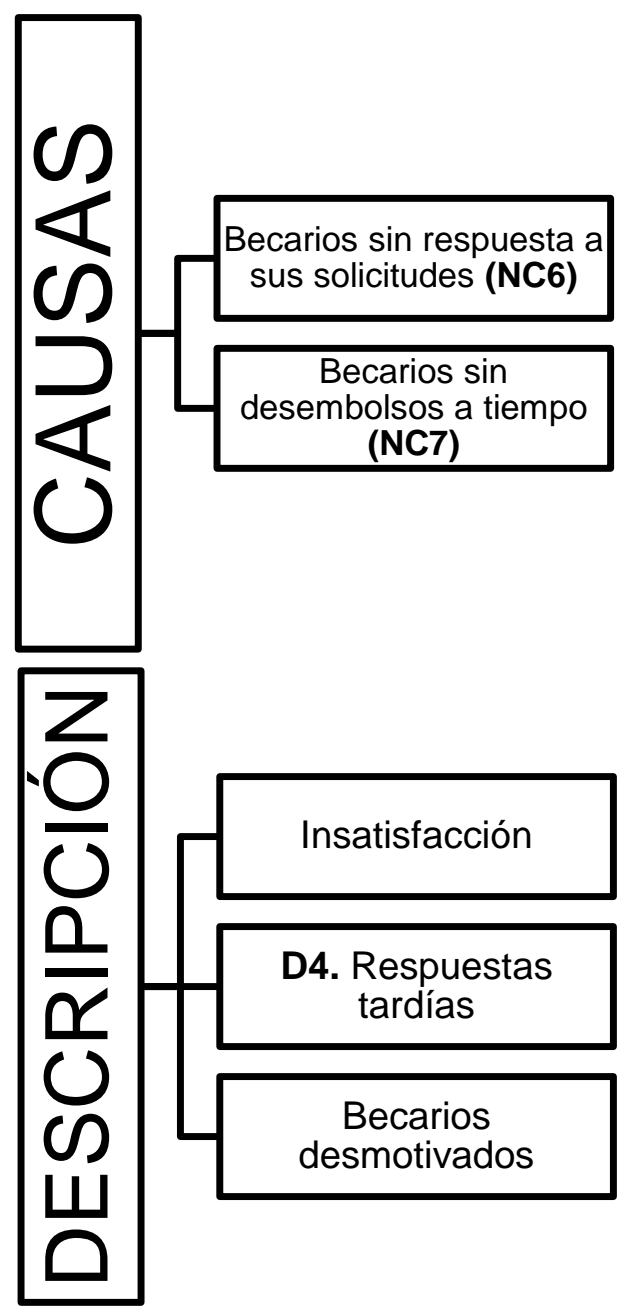




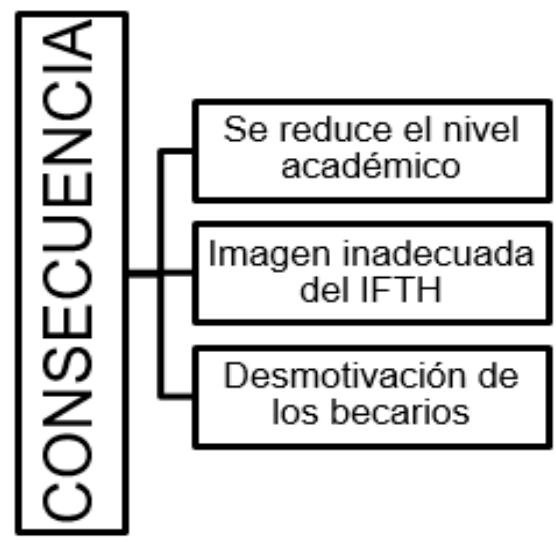

Figura 8. Los tiempos en resolver dudas, inquietudes o desembolsos de manera online. (P4). Nota: $(P)=$ Problema, $(D)=$ Descriptor, $(\mathrm{NC})=$ Nudo Crítico

\section{Paso 4: Selección de causas identificadas en el árbol explicativo}

\section{Falta de presupuesto (NC1)}

2. Deficiente selección de personal (NC2)

3. Personal de becas sin capacitación (NC3)

4. Falta de planificación (NC4)

5. No existe un estudio para determinar necesidades de los becarios (NC5)

6. Becarios sin respuesta a sus solicitudes (NC6)

7. Becarios sin desembolsos a triempo (NC7)

(NC) $=$ Nudo Crítico 
David Sebastián Flores-Romero; Hugo Jaime Avendaño-Fajardo; Mireya Magdalena Torres-Palacios

\section{Paso 5: Sellección de frentes de ataque o nudos críticos}

Tabla 3.

Selección de frentes de ataque o nudos críticos. Nota: (A) Alto, (M) Medio, (B) Bajo.

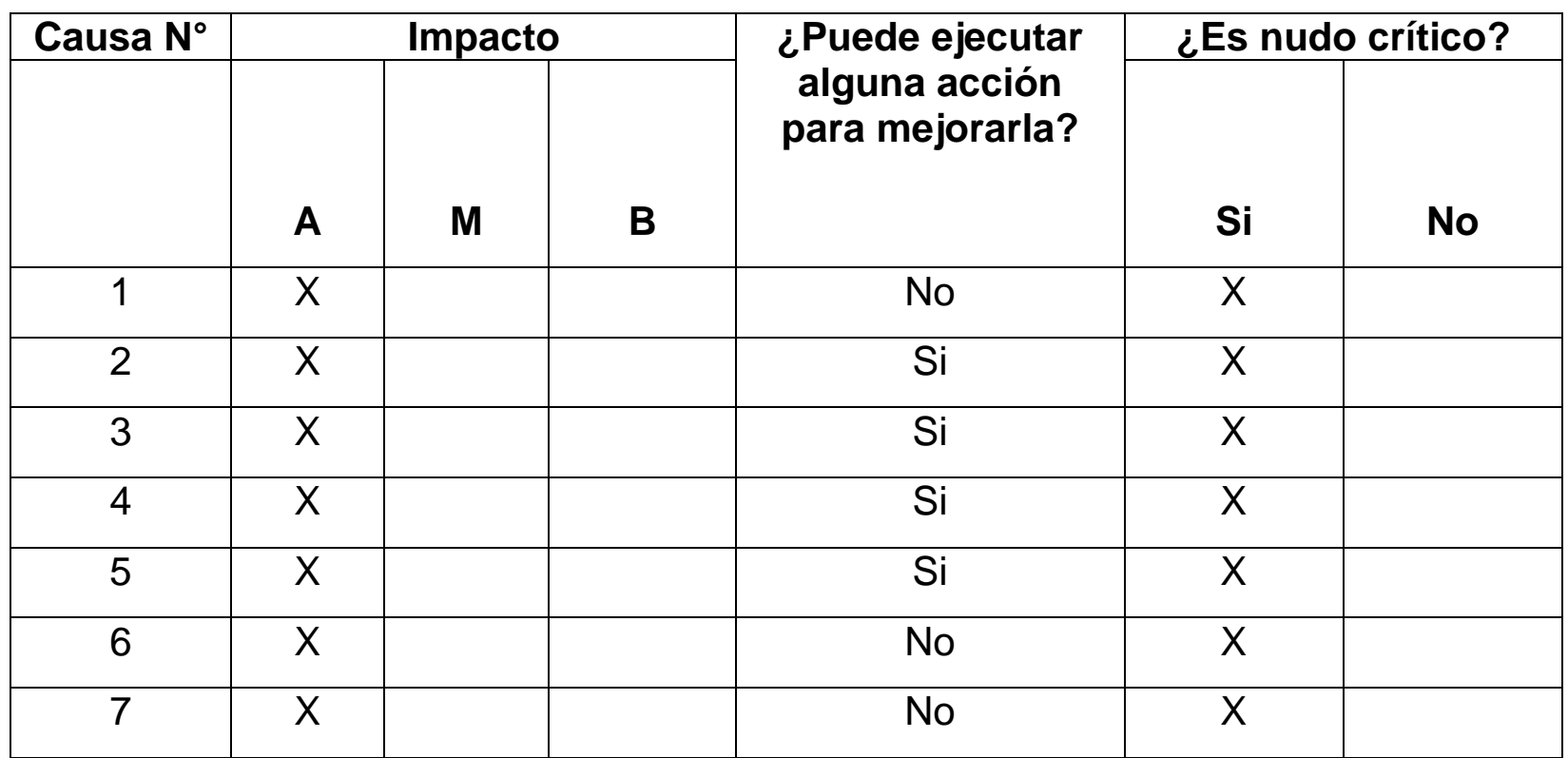

\section{Paso 6: Diseño de demandas de operación}

\section{Tabla 4.}

Diseño de demandas de operación.

\begin{tabular}{|c|c|c|c|}
\hline Nudo crítico & ¿Qué hacer? & ¿Quién hace? & ¿Quién coopera? \\
\hline Deficiente selección & Diseñar e & Departamento de & Ministerio de \\
de personal (NC2) & implementar & Talento Humano & economía y finanzas \\
& $\begin{array}{c}\text { un sistema de } \\
\text { selección }\end{array}$ & & \\
& $\begin{array}{c}\text { adecuado a las } \\
\text { necesidades }\end{array}$ & & \\
& Programa de & Departamento de & Coordinadores \\
& capacitación & Talento Humano & Zonales \\
\hline Personal de becas & & & \\
\hline
\end{tabular}


Revista Arbitrada Interdisciplinaria KOINONIA

Año 2020. Vol V. №3. Especial: Administración

Hecho el depósito de Ley: FA2016000010

ISSN: 2542-3088

FUNDACIÓN KOINONIA (F.K). Santa Ana de Coro. Venezuela.

David Sebastián Flores-Romero; Hugo Jaime Avendaño-Fajardo; Mireya Magdalena Torres-Palacios

\begin{tabular}{|c|c|c|c|}
\hline $\begin{array}{c}\text { (NC3) } \\
\text { (NC4) }\end{array}$ & $\begin{array}{c}\text { Planes de acción } \\
\text { departamentales }\end{array}$ & $\begin{array}{c}\text { Responsable de cada } \\
\text { departamento }\end{array}$ & $\begin{array}{c}\text { Departamento de } \\
\text { planificación }\end{array}$ \\
\hline $\begin{array}{c}\text { No existe un estudio } \\
\text { para determinar } \\
\text { necesidades de los } \\
\text { becarios (NC5) }\end{array}$ & $\begin{array}{c}\text { Aplicación de } \\
\text { instrumentos de } \\
\text { investigación para } \\
\text { conocer las } \\
\text { necesidades de los } \\
\text { estudiantes }\end{array}$ & $\begin{array}{c}\text { Departamento de } \\
\text { Comunicación }\end{array}$ & $\begin{array}{c}\text { Departamento de } \\
\text { Talento Humano }\end{array}$ \\
& & \\
\hline
\end{tabular}

Fuente: Matus (2007).

\section{Paso 7: Definición de responsables por las demandas de operaciones}

\section{Tabla 5.}

Definición de responsables por las demandas de operaciones. Nota: $(\mathrm{DOp})=$ Demanda de operación.

\begin{tabular}{|c|c|}
\hline Operaciones & Responsable \\
\hline $\begin{array}{c}\text { DOp1. Diseñar e implementar } \\
\text { un sistema de selección } \\
\text { adecuado a las } \\
\text { necesidades }\end{array}$ & Departamento de Talento Humano \\
\hline DOp2. Programa de capacitación & Departamento de Talento Humano \\
\hline DOp3. Planes de acción departamentales & Responsable de cada departamento \\
investigación para conocer las necesidades \\
de los estudiantes
\end{tabular}


David Sebastián Flores-Romero; Hugo Jaime Avendaño-Fajardo; Mireya Magdalena Torres-Palacios

\section{Paso 8: Presupuesto del plan}

\section{Tabla 6.}

Presupuesto del plan.

\begin{tabular}{|c|c|c|c|}
\hline $\begin{array}{c}\text { Demanda de } \\
\text { operación }\end{array}$ & Costo en trabajo & Aporte al IFTH & $\begin{array}{c}\text { Costo total en } \\
\text { dinero }\end{array}$ \\
\hline $\begin{array}{c}\text { Diseñar e } \\
\text { implementar } \\
\text { un sistema de } \\
\text { selección } \\
\text { adecuado a las } \\
\text { necesidades }\end{array}$ & 20 horas & USD \$ 500 & USD \$ 500 \\
\hline $\begin{array}{c}\text { Programa de } \\
\text { capacitación }\end{array}$ & 100 horas & USD \$ 3.000 & USD \$ 3.000 \\
\hline $\begin{array}{c}\text { Planes de acción } \\
\text { departamentales }\end{array}$ & 20 horas & USD \$ 500 & USD \$ 500 \\
\hline $\begin{array}{c}\text { Aplicación de } \\
\text { instrumentos de } \\
\text { investigación para } \\
\text { conocer las } \\
\text { necesidades de los } \\
\text { estudiantes }\end{array}$ & 50 horas & USD \$ 1.000 & USD \$ 1.000 \\
\hline \multicolumn{2}{|r|}{} & & USD \$ 5.000 \\
\hline
\end{tabular}

Fuente: Matus (2007). 


\section{Paso 9: Identificación de actores relevantes y su motivación frente al plan}

\section{Tabla 7.}

Identificación de actores relevantes y su motivación frente al plan. Nota: $(D O P)=$ Demanda de operación $(\mathrm{V})=$ Valor $(\mathrm{A})=$ Alto $(+)=$ Apoyo

\begin{tabular}{|c|cr|cr|cc|cc|cc|}
\hline DOP & \multicolumn{2}{|l|}{ DOp 1 } & \multicolumn{2}{|l|}{ DOp 2 } & \multicolumn{2}{|l|}{ DOp 3 } & \multicolumn{2}{|c|}{ DOp 4 } \\
ACTORES & V & I & V & I & V & I & V & I \\
\hline Departamento de Talento Humano & $\mathrm{A}$ & + & $\mathrm{A}$ & + & $\mathrm{A}$ & + & $\mathrm{A}$ & + \\
\hline Responsable de cada departamento & $\mathrm{A}$ & + & $\mathrm{A}$ & + & $\mathrm{A}$ & + & $\mathrm{A}$ & + \\
\hline Departamento de Comunicación & $\mathrm{A}$ & + & $\mathrm{A}$ & + & $\mathrm{A}$ & + & $\mathrm{A}$ & + \\
\hline
\end{tabular}

\section{Paso 10: Selección de trayectorias (momento táctico)}

\section{Tabla 8.}

Selección de trayectorias (momento táctico).

\begin{tabular}{|l|l|}
\hline & \multicolumn{1}{|c|}{ Demanda de operación } \\
\hline A & Diseñar e implementar un sistema de selección adecuado a las necesidades \\
\hline B & Programa de capacitación \\
\hline C & Planes de acción departamentales \\
\hline D & $\begin{array}{l}\text { Aplicación de instrumentos de investigación para conocer las necesidades de los } \\
\text { estudiantes }\end{array}$ \\
\hline
\end{tabular}


David Sebastián Flores-Romero; Hugo Jaime Avendaño-Fajardo; Mireya Magdalena Torres-Palacios

Para establecer la ruta crítica de las demandas de operación, se utilizó el software POM/QM en la parte de gestión de proyectos (PERT/CPM), donde se determinó el tiempo del plan estratégico en 8 meses como se observa a continuación:

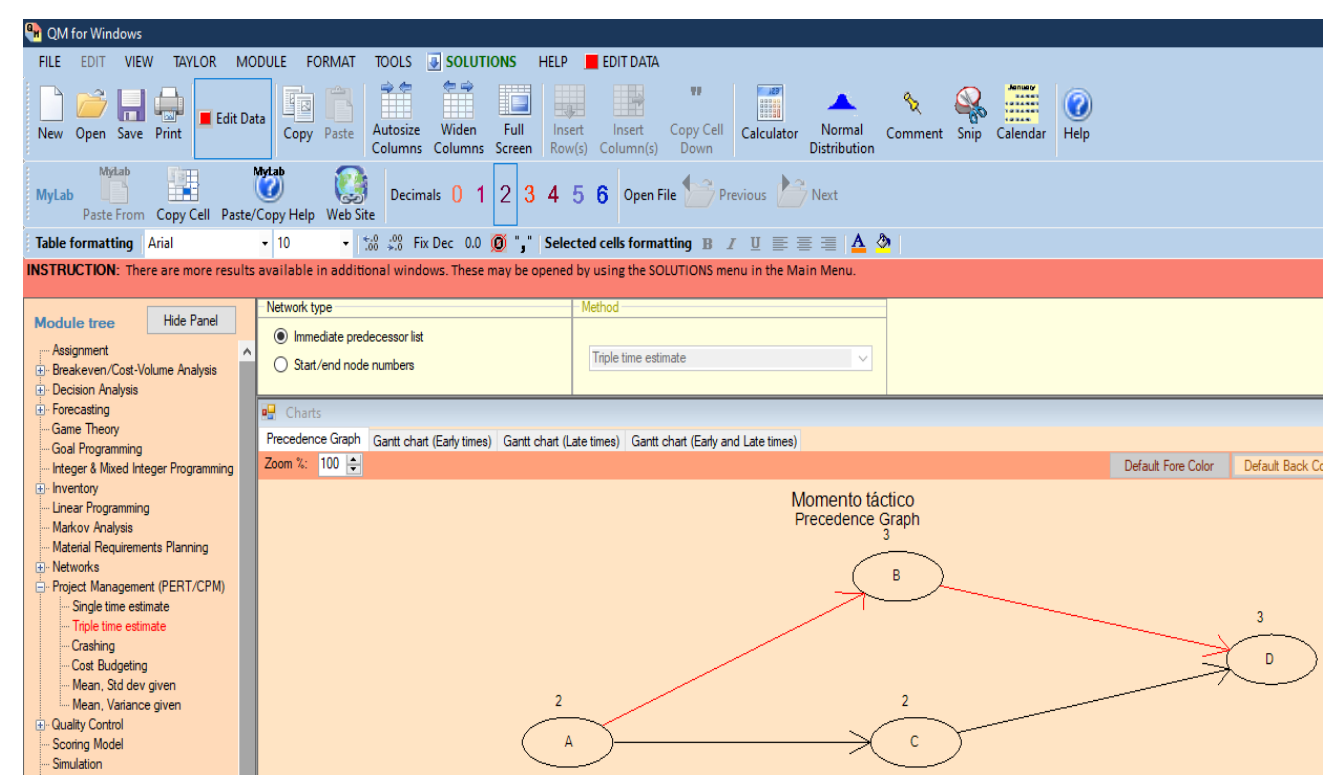

Figura 9. Gestión de proyectos (PERT/CPM). 


\section{Paso 11: Análisis de vulnerabilidad del plan}

Tabla 9. Análisis de vulnerabilidad del plan.

\begin{tabular}{|c|c|c|c|}
\hline Condicionante letal & Impacto & ¿De quién depende? & Acción alterna \\
\hline $\begin{array}{c}\text { Diseñar e implementar un } \\
\text { sistema de selección } \\
\text { adecuado a las necesidades }\end{array}$ & Alto & $\begin{array}{c}\text { Departamento de } \\
\text { Talento Humano }\end{array}$ & SENESCYT \\
\hline Programa de capacitación & Alto & $\begin{array}{c}\text { Departamento de } \\
\text { Talento Humano }\end{array}$ & SENESCYT \\
\hline $\begin{array}{c}\text { Planes de acción } \\
\text { departamentales }\end{array}$ & Alto & $\begin{array}{c}\text { Responsable de cada } \\
\text { departamento } \\
\text { Departamento de } \\
\text { Comunicación }\end{array}$ & SENESCYT \\
\hline $\begin{array}{c}\text { Aplicación de instrumentos de } \\
\text { investigación para conocer las } \\
\text { necesidades de los } \\
\text { estudiantes }\end{array}$ & Alto & SENESCYT \\
\hline
\end{tabular}

Fuente: Matus (2007).

Paso 12: Diseño del sistema de petición verbal y rendición de cuentas

Tabla 10.

Diseño del sistema de petición verbal y rendición de cuentas

\begin{tabular}{|l|l|l|}
\hline \multicolumn{3}{|c|}{ Rendición de cuentas } \\
\hline Nombre del responsable: & \multicolumn{2}{|l|}{} \\
\hline Período de la cuenta: & Desde: \\
\hline Fecha de la cuenta: & \\
\hline Operación evaluada: & \\
\hline \multicolumn{1}{|c|}{ Supuestos letales al momento de la cuenta: } \\
\hline Cumplidos: & Incumplidos: & Semicumplidos: \\
\hline Resultados previstos: & Resultados obtenidos & Causas \\
\hline & & \\
\hline & & \\
\hline Decisión : & & \\
\hline Responsable: & & \\
\hline
\end{tabular}

Fuente: Matus (2007). 


\section{CONCLUSIONES}

El triunfo de la planificación estratégica situacional radica en el dominio de antelación, la decisión y la reacción pertinente del cambio, respaldando sus hechos con un procedimiento o plan lógico como es el método altadir de planificación popular, estableciendo así los objetivos del instituto y la definición de los procesos adecuados para alcanzarlos. La PES ayudó a fijar prioridades, permitiendo concentrarse en las fortalezas y debilidades del instituto, ayudando a solventar los problemas de cambios en el entorno interno y externo, depende de ella que se logren los objetivos organizacionales con éxito. El IFTH en un tiempo de 10 meses puede mejorar todos los servicios en cuanto a becas para poder satisfacer las necesidades de los becarios que se encuentran al momento inconformes por temas de insatisfacción de los becarios.

\section{FINANCIAMIENTO}

No monetario.

\section{AGRADECIMIENTO}

Al Instituto de Fomento al Talento Humano del Ecuador, por permitir el desarrollo de esta investigación. 


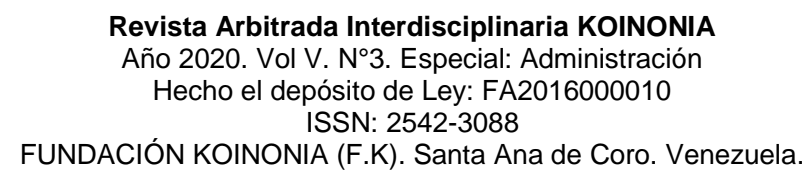

David Sebastián Flores-Romero; Hugo Jaime Avendaño-Fajardo; Mireya Magdalena Torres-Palacios

\section{REFERENCIAS CONSULTADAS}

Argudo-Tello, K. J., Erazo-Álvarez, J. C., \& Narváez-Zurita, C. I. (2019). Evaluación de Control Interno en Riesgos Estratégicos para la Dirección de Planificación de la Universidad de Cuenca. [Evaluation of Internal Control in Strategic Risks for the Planning Direction of the University of Cuenca]. Revista Arbitrada Interdisciplinaria Koinonía, 1(4), 67-96. http://dx.doi.org/10.35381/r.k.v4i1.372

Armijo, M. (2011). Planificación estratégica e indicadores de desempeño en el sector público. [Strategic planning and performance indicators in the public sector]. Instituto latinoamericano y del caribe de planificación económica y social. Recuperado de https://n9.cl/m5jw

Ávila, E., Erazo, J. C., Narváez, C. I., \& Erazo, C. A. (2019). Estrategias de marketing digital 2.0 para la generación de ingresos en Pymes de servicios. Revista Arbitrada Interdisciplinaria Koinonía, 1 (5 edición especial), 187-214. https://doi.org/10.35381/cm.v5i1.264

Calle-Abril, D. R., Erazo-Álvarez, J. C., \& Narváez-Zurita, C. I. (2020). Estrategias de mediación y solución de conflictos para el sector industrial de pinturas [Mediation and conflict resolution strategies for the paint industry]. Revista Arbitrada Interdisciplinaria Koinonía, 10(5), 514-544. http://dx.doi.org/10.35381/r.k.v5i10.704

Criollo-Aguirre, M. J., Erazo-Álvarez, J. C., \& Narváez-Zurita, C. I. (2019). Estrategias de Marketing y posicionamiento de marca para el sector artesanal textil. [ Marketing strategies and brand positioning for the textile artisan sector]. Cienciamatria, 245270. https://doi.org/10.35381/cm.v5i1.266

Gutiérrez, J. M., Alizo, M. A., Morales, M., \& Romero, J. (2016). Planificación estratégica situacional: Perspectiva de una unidad científica universitaria [Situational strategic planning: Perspective of a university scientific unit]. Revista venezolana de gerencia, 21(76), 607-626.

Instituto de Fomento al Talento Humano. (2016). Plan estratégico 2016 - 2017 [Strategic plan 2016 - 2017.]. Recuperado de https://n9.cl/nauc

Instituto de Fomento al Talento Humano. (2020). Fomento académico-becas. [Academic promotion - scholarships.]. Recuperado de https://n9.cl/00g8

Matus, C. (2007). MAPP Método Altadir de Planificación Popular. [MAPP Altadir Method of Popular Planning]. 1 era edición. Buenos Aires: Lugar Editorial. 
Ossorio, A. (2003). Planeamiento estratégico. [Strategic planning]. Buenos Aires: INAP. Recuperado de: https://n9.cl/xe9l

Reyes, P. E., Narváez, C. I., Erazo, J. C., \& Giler, L. V. (2020). Configuración del impuesto a la patente municipal con base al ingreso de las actividades económicas. Caso: GAD Municipal de Pucará - Ecuador. [Configuration of the municipal patent tax based on income from economic activities. Case: GAD Municipal de Pucará Ecuador]. Revista Espacios, 41(21), 197-211.

Rivera, F. J. (2011). Argumentación y construcción de validez en la planificación estratégica situacional de Matus [Argumentation and construction of validity in Carlos Matus' Situational Strategic Planning]. Cadernos de Saúde Pública, 27(9), 1847-1858. https://doi.org/10.1590/S0102-311X2011000900018.

Salazar, D., \& Romero, G. E. (2006). Planificación. ¿Éxito Gerencial? [Planning for Managerial Success?]. Multiciencias, 6(1), 1-17. 